\title{
Belphégor
}

\section{Par tous les moyens : territoire du milieu et champ de forces}

\section{Paul Bleton}

\section{(2) OpenEdition}

\section{Journals}

\section{Electronic version}

URL: http://journals.openedition.org/belphegor/1037

DOI: 10.4000/belphegor.1037

ISSN: 1499-7185

\section{Publisher}

LPCM

\section{Electronic reference}

Paul Bleton, «Par tous les moyens : territoire du milieu et champ de forces », Belphégor [Online], 15-2 | 2017, Online since 23 November 2017, connection on 02 May 2019. URL : http:// journals.openedition.org/belphegor/1037; DOI : 10.4000/belphegor.1037

This text was automatically generated on 2 May 2019.

\section{(c) () $\odot$}

Belphégor est mis à disposition selon les termes de la Licence Creative Commons Attribution - Pas d'Utilisation Commerciale - Pas de Modification 4.0 International. 


\title{
Par tous les moyens : territoire du milieu et champ de forces
}

\author{
Paul Bleton
}

1 Héritons de cette double hypothèse qu'il existerait dans le domaine français une production romanesque intermédiaire comparable à celle de la littérature middlebrow anglo-saxonne, corrélée à des pratiques culturelles moyennes, le territoire du milieu; et, même si la critique le laisse encore largement inexploré alors même qu'il est largement connu du public, que ce territoire du milieu présupposerait, directement ou non, explicitement ou non, que le régime médiatique ait pu y imposer une reconfiguration systémique à la fiction narrative, elle-même bien plus ancienne. Avec quelles précautions de méthode cette problématique du middlebrow pourrait-elle devenir pertinente pour le domaine français?

2 Pour des raisons de format, et même si elles sont toutes nécessaires, c'est ailleurs que seront franchies les étapes préalables consistant à rappeler l'histoire de la problématique originale en Grande-Bretagne et aux États-Unis, à repérer la conceptualisation du moyen dans la tradition sociologique française et à préciser la définition intensionnelle d'un concept qui n'est pas en usage dans la critique de langue française. Voire, alors même que ce n'est pas seulement la définition intensionnelle (ou plutôt celle de son équivalent français) mais son extension qui est encore floue et qu'il faudrait commencer par une première approximation extensionnelle de la notion appliquée au roman français, c'est aussi ailleurs que sera exposé. On peut toutefois mentionner un premier résultat d'une telle enquête préalable: l'approximation extensionnelle de la notion, l'approche cartographique du territoire du milieu décrit de proche en proche, d'un échantillon à l'autre, par repérage de frontières, évaluation de son étendue et de son altimétrie en matière de légitimité littéraire, révèlent que la singularité de chaque auteur fait rapidement croitre le nombre de variables. Tendant à rapidement augmenter, elles brouillent la carte en proportion.

3 Sans abandonner l'objectif descriptif, cet inconvénient conduirait à proposer une approche alternative mais complémentaire, à passer d'un modèle cartographique du territoire à un modèle physique des interactions : en généralisant l'effet d'attraction 
centripète repéré dans les évaluations entrecroisées et en identifiant l'effet d'attraction centrifuge produit par les territoires environnants.

\section{Célébrité}

4 Alors même que la question initiale de ce numéro de Belphégor identifiait trois classes de romans, elle tendait à les rabattre sur une problématique dualiste, opposant les romans dont études littéraires et paralittéraires se préoccupent et ceux qui ne sont pas décrits, dévalués par l'adjectif «moyen». Or la complexité entraperçue en commençant à cartographier le territoire du milieu français incite à douter de la pertinence de quelque dualisme que ce soit. La vue synoptique (Übersicht), une idée de Ludwig Wittgenstein, tirée $\mathrm{du} \S 122$ de ses Investigations philosophiques, propose d'éclairer autrement tout groupe de phénomènes nombreux en les disposant de telle sorte que soit provoquée une modification dans notre perception et notre compréhension, pour tenter d'échapper aux ornières, aux habitudes, aux rigidités et aux confusions spontanées de la pensée, pour mettre en vedette ressemblances et différences évidentes et moins évidentes, pour éclaircir les liens entre phénomènes... Plutôt que de subir l'adjectif « moyen » comme une marque d'infamie, mieux vaudrait proposer que la question de l'évaluation ne se résume pas à une altimétrie cartographiable. Que cette activité soit un des universaux cognitifs de l'acte de lecture, voire de la vie en société, ne doit pas évacuer des questions comme "qui évalue et selon quels critères?", "quelles tractations pour parvenir à quel consensus évaluatif?». Ce qui devrait permettre une première vue synoptique; l'hypothèse étant qu'elle se ferait à partir de la refonte médiatique de la notion de célébrité.

5 La forte connotation dépréciative de "culture moyenne " sous la plume de prescripteurs littéraires ne date pas d'hier. Voici une phrase tirée d'un autre éreintement en règle que le critique mondain Augustin Filon faisait d'un roman de Georges Ohnet dans La Revue bleue ( $n^{\circ} 23,7$ Déc. 1889) : «Quant au style, vous le connaissez : il semble qu'un correcteur patient en ait soigneusement émondé tout ce qui pourrait étonner, arrêter ou faire penser un bourgeois de culture moyenne, sa «dame» ou sa "demoiselle».» (p. 729) ${ }^{\mathrm{ii}}$. Considérons Ohnet comme un symptôme, celui d'un moment historique de l'édition française, celui de la réaffirmation par les revues d'avant-garde à partir de $1885^{\text {iii }}$ d'une prééminence esthétique et éthique de la création littéraire en littérature contre la conception marchande de l'édition ${ }^{\text {iv }}$. La Revue bleue, née en 1863, se devait de sentir le vent, quitte à ce que Filon propose une conception de la moyennisation par soustraction, par ascèse, mise en œuvre pour de mauvais motifs, non pas esthétiques mais éthiques, qui signent l'assujettissement de la création artistique aux bonnes mœurs ${ }^{v}$. Cette ancienneté rend-elle pour autant la connotation négative indépassable?

6 Renversons la perspective pour mettre en lumière les dynamiques multiformes de l'évaluation en culture moyenne. En France, le mécanisme de distinction attribuant à la cour le statut de source et d'arbitre du goût ne s'est que très lentement évanoui - a-t-il même disparu ? En matière de littérature romanesque, la posture critique aristocratique a longtemps survécu à la forme de société hiérarchisée de l'Ancien régime. Moteur à deux temps, ce mécanisme critique repose sur la convergence du consensus et la divergence de la démarcation. Le consensus est celui des élites. Il est obtenu par sédimentation, il se configure en un canon littéraire national auquel contribuent non seulement l'aréopage des spécialistes de l'institution littéraire (prescripteurs, voire éditeurs et auteurs) mais 
aussi l'École (de nouvelles générations peuvent actualiser de grandes voix du passé, se reconnaître dans des œuvres ainsi pérennisées, comprendre la révérence qui leur est due). Ici, le livre louche du côté de la consécration qui pérennise une légitimation préalable. La démarcation est celle de l'esprit supérieur. Ce dernier vise à dépasser un tel consensus - opération para-doxale, donc, pour peu qu'un consensus sur le canon ait été préalablement atteint et puisse être tenu pour une doxa, fût-ce dans un cercle restreint. Selon que la démarche est celle d'un auteur ou d'un prescripteur, comparable, le sens de la démarcation n'est pas pour autant identique. Lorsque le créateur veut faire école en se démarquant, il définit le geste de rupture propre à l'avant-garde ; affirmant sa solitude ; le prescripteur, lui, établit la communauté des happy-few amateurs de telle œuvre rare. Toutefois, la progressive mise en place d'un régime médiatique, tout en s'accommodant de cette posture aristocratique alliant consensus des élites et démarcation de l'esprit supérieur, a non seulement expérimenté d'autres modes d'attribution de la valeur littéraire mais il les a aussi fait coexister : il adapte les marqueurs de fortune traditionnels de l'institution littéraire, les fait converger ou diverger avec ceux du marché et avec ceux propres à la culture médiatique.

7 Depuis le Goncourt, qui devait initialement servir de contrepoids aux prix décernés par l'Académie française, les prix littéraires sont un important marqueur de fortune. De plus en plus nombreux et divers, tous n'ont certes ni la même distinction, ni le même empan culturel, ni la même incidence sur les ventes mais tous permettent à l'actualité de la création romanesque de s'inscrire dans l'actualité journalistique ou, au moins, de pointer du doigt tel roman dans la pléthore de ceux qui paraissent en même temps. Afin de donner une idée de cette diversité, si l'on se restreint au seul roman de guerre, que l'on se souvienne du Goncourt attribué à Capitaine Conan (1934) de Roger Vercel, de l'Interallié au Commandant Watrin (1956) d'Armand Lanoux, du Renaudot aux Combattants du petit bonheur (1977) d'Alphonse Boudard, du Prix littéraire des lectrices de Elle à Couvre-feux (1983) de Clarisse Nicoidski... Par ailleurs, même s'ils sont littéraires, il arrive que ces prix soient très directement branchés sur l'Histoire en marche, sorte de contrepoints à l'actualité. En 1953, en pleine affaire Henri Martin ${ }^{\text {vi }}$, le Grand Prix du roman de l'Académie française est accordé au cinquième volume de La Nuit indochinoise cycle romanesque de Jean Hougron, alors en cours de parution!

Non plus dans l'institution littéraire mais dans l'appareil éditorial, ce sont les rééditions et les traductions qui servent de marqueurs de fortune. En huit décennies, La Madone des sleepings a connu une demi-douzaine de rééditions dont une chez France loisirs et trois en format poche $e^{\text {vii }}$; idem pour Le Maître de forges en un siècle. L'Escadron blanc (1931) de Joseph Peyré, une douzaine entre 1931 et 1977 ; idem pour Chiens perdus sans collier de Gilbert Cesbron entre 1954 et 1981; une quinzaine pour Maria Chapdelaine de Louis Hémon, plus d'une vingtaine pour L'Atlantide et, depuis 1958, une trentaine pour Le Lion de Kessel. C'est évidemment grâce à sa réédition en 1976 que L'Officier sans nom (1941) de Guy des Cars devait vendre un demi-million d'exemplaires, pas grâce à son édition originale en in- $8^{\circ}$ à Cannes chez un imprimeur ni même grâce à son " Goncourt de zone libre ». Les rééditions peuvent aussi être l'occasion de découvrir de nouveaux marchés: si les différentes éditions de Flammarion ont bien conservé son caractère populiste au Feu d'Henri Barbusse, voire cherché à le rendre accessible par le coût ${ }^{\text {viii }}$, ce roman est aussi un favori des collections de format club ${ }^{\mathrm{ix}}$ et sert parfois de matériau à un beau livre illustréx.

9 L'évaluation des lectrices rendue publique reste indirecte dans un cas comme le Grand Prix des lectrices de Elle mais anonyme et directe dans le cas d'un succès marchand. 
Puisque nombre de romans mentionnés jusqu'ici ont été des succès de vente, pour caractériser le territoire du milieu il serait opportun de tenir le statut de best-seller comme un critère pertinent ${ }^{\mathrm{xi}}$, chacun dans la singularité de son histoire servant de jauge pour comparer des romans apparentés mais moins fortunés.

Ce n'est pas seulement à titre symbolique que l'on peut considérer l'adaptation d'un roman comme une évaluation effectuée par la culture médiatique ; une adaptation pour l'écran provoque généralement un accroissement ou un regain d'intérêt pour le roman adapté. En se restreignant à quelques romans mentionnés jusqu'ici, soulignons la fortune d'Ohnet plusieurs fois adapté, parfois plusieurs fois pour un même titre - comme Le Maître de forges par Henri Pouctal en film muet en 1912, par Abel Gance, une première fois directement en 1933 et une deuxième fois en supervisant Fernand Rivers en 1948. Non content de cette réussite française, il est aussi porté à l'écran à l'étranger avec Il Padrone delle ferriere (1959) d'Anton Guilio Majano mais aussi les Serges Panine de Wray B. Physioc (1915) et Evgueny Bauer (1916), respectivement aux États-Unis et en Russie. Si qualitativement Chiens perdus sans collier de Cesbron que Jean Delanoy porte à l'écran en 1955 ou Week-end à Zuydcoote de Merle qu'Henri Verneuil adapte en 1964 n'ont pas à rougir, ils restent pâlichons devant La Bataille de Farrère - roman adapté par Pierre Frondaie pour à la scène, au théâtre Antoine en 1921, adaptation elle-même remaniée pour la radio, à la BBC en anglais, en 1912 et 1936, en français à Radio-Canada en 1939 $9^{\text {xii }}$ et à la Radio nationale en 1952, en opéra sur une musique d'André Gailhard à la GaîtéLyrique en 1931, à l'écran par Edouard Émile Violet et Sessue Hayakawa en 1923 et deux fois par Nicolas Farkas en 1933 (en français puis en anglais, avec la même réalisation et les mêmes acteurs, seule Merle Oberon se substituant à Annabella), voire en BD, dans FranceSoir en septembre-octobre 1959, avec des illustrations d'Andréas Rosenberg. La transmédiatisation, on l'a vu, peut se faire transnationale. Non seulement L'homme qui assassina est-il deux fois adapté à l'écran en France (par Henri Andreani en 1913 et par Kurt Bernhart et Jean Tarride en 1933) mais aussi en 1920 par George Fitzmaurice sous le titre The Right to Love. Si le prolifique des Cars est relativement peu porté à l'écran en France ${ }^{\text {xiii }}$ La Brute était devenu The Green Scarf (1954) devant la caméra britannique de George More O'Ferrall, Cette étrange tendresse (1960) Extraña ternura (1964) devant celle, argentine, de Daniel Tinayre, et Les Filles de joie (1959) Las Hermanas (1962) toujours par Tinayre. Pour La Bataille, c'est l'adaptation théâtrale de Frondaie qui passait à l'écran; mais il peut aussi y avoir d'autres formes de transmédiatisation en plus de deux étapes. Ainsi Rapa-Nui d'Armandy a-t-il d'abord paru sous forme de roman en 1923, puis été adapté à l'écran par Mario Bonnard en 1928, film qui à son tour a été novélisé dans la collection Cinéma-bibliothèque de Tallandier la même année.

11 Nouvelle tendance dans les années récentes, l'évaluation effectuée par la culture médiatique se déporte sur les auteurs, pipolise best-sellers et romanciers apparentés à la culture médiatique, comme Philippe Labro, journaliste et homme de médias, réalisateur, adaptateur, parolier, romancier (une vingtaine de romans publiés en une trentaine d'années) récompensé par les prix Interallié et Gutenbergiv. Toutefois, attention: un auteur fortement sollicité par les médias ne relève pas ipso facto du territoire du milieu. Certes on peut se poser la question pour Alexandre Jardin, auteurs de plusieurs best-sellers , lauréat des Prix du premier roman (en 1986) et Fémina (en 1988), malgré ses romans mythifiant et démystifiant son arbre généalogique en un mouvement pendulaire. En revanche, La Gloire de l'Empire (1971) a beau être une uchronie devenue best-seller, outre que cela en fait une rareté, même si son Grand prix du roman de l'Académie française a 
permis à Jean d'Ormesson de devenir académicien en 1972, biographié depuis ${ }^{\mathrm{xv}}$, ce sont ses romans remettant en cause la narrativité classique qui le placent hors du territoire du milieu.

12 À ce succinct relevé, on se contentera ici d'ajouter au passage les effets du croisement de ces critères d'évaluation - aussi bien la relance des ventes du roman par une adaptation, l'optimisation du succès d'un film (parfois lui-même adapté) par une novélisation parasite, l'incidence d'un prix littéraire sur les ventes ${ }^{\mathrm{xvi}}$ que les mises en garde imposée par toute situation complexe $e^{\text {xvi }}$ - de même que l'accroissement par le nombre et par le succès de la part de la traduction dans les best-sellers ${ }^{\text {xviii }}$ ou la sociabilité seconde des fans (des clubs de lecture aux groupes de discussion sur l'internet).

13 Tel serait la première vue synoptique permise par le modèle physique des interactions venant enrichir le modèle cartographique du territoire : la célébrité. Bien entendu, la célébrité n'est pas simplement l'affaire de la culture moyenne ; elle n'en a pas moins été redéfinie par le régime médiatique, glissant en son centre pour en occuper la place de valeur-noyau. Ainsi le temps visé par la célébrité avant le régime médiatique était-il l'immortalité, version glorifiée du temps long de la lecture et, surtout, de l'écriture ; avec la culture médiatique, le temps de la célébrité est l'instantanéité, consommée immédiatement - ce qui en altère complètement la fonction et la signification. On le voit, elle émane de sources diverses et instaure des hiérarchies multiples, enchevêtrées, parfois convergentes mais pas toujours.

\section{Effets attracteurs}

Si le territoire du milieu est un champ de forces et si décrire les marqueurs de fortune mesurant la célébrité est nécessaire, ce n'est toutefois pas suffisant. Des forces relevant non plus des agents évaluateurs mais émanant des territoires environnants, des autres classes de romans, y produisent en effet des effets d'attraction, structurants ou embrouilleurs : telle serait notre seconde vue synoptique.

Revenons tout d'abord à la question initiale. Tournant le dos au roman de «haute culture », le roman de culture moyenne résidant dans le territoire du milieu puiserait ses ressources narratives et pragmatiques dans la fiction populaire et le ferait pour aborder des « questions sérieuses ». Si l'on comprend bien qu'il refuse le « modernisme sceptique, réflexif et autoréférentiel ", à quelles influences se livre donc le roman du territoire du milieu pour devenir sérieux ? Examinons-en quatre.

16 Au-delà de l'effet que continue d'exercer le mimétisme réaliste, sans doute le plus important attracteur dans ce territoire, l'œuvre de Gilbert Cesbron précise l'une des stratégies d'instauration du sérieux. Quoi de plus sérieux que cet auteur qui pendant une trentaine d'années a fictionnalisé des problèmes d'actualité sous un regard chrétien plein de compassion, séduisant un public nombreux - une demi-douzaine de ses romans ont dépassé le million d'exemplaires, Chiens perdus sans collier (1954) arrivant même à 4 millions (l'histoire d'enfants issus de milieux défavorisés qu'un juge saisi de leurs dossiers après qu'ils aient commis des délits tente d'aider) ? Comme un casuiste, en une variante du roman de mœurs, le romancier-moraliste singularise un problème de société et fait part de son jugement de valeurs, plutôt consensuel, à la fois par le déroulement de l'intrigue et par des aphorismes servant à boutonner la narration. La prose de Cesbron balance entre sérieux et jeu. Dans C'est Mozart qu'on assassine (1966), un roman sur le 
divorce ou tend à prévaloir le point de vue de Martin, le fils unique de 7 ans partagé entre ses deux parents, je glane des vérités bien senties comme «Les enfants uniques sont souvent ainsi élevés qu'ils se dédoublent, l'un ne cessant de surveiller l'autre et de le protéger. » (p. 18) ou « On est de son enfance comme on est d'un pays. » (p. 46) mais aussi des fantaisies faisant tressauter le langage (expression campagnarde comme dormir comme une pionce, p. 88; idiolecte d'une famille de médecins parlant d'« un turbigo dans le pataf " pour «un suppositoire dans le derrière ", p. 89 ; mnémotechnique d'une pédagogie à l'ancienne pour la liste des départements, leurs préfectures et souspréfectures : «Un homme avait une soif de l'Yonne. Il Joigny le Sens à la parole et se dit « Tonnerre, Avallon! » ", p. 92 ; à quoi répondent les à-peu-près du petit garçon, « Le café est éclairé au néant ", p. 103). Mais la narration au mimétisme transparent, entièrement au service de l'argumentation éthique, tient étroitement en lisière aphorismes mesurés ou définitifs et écarts poétiques. Tout aussi mimétique, transparente et assujettie à un moralisme bienveillant, le Monsieur Blot de Daninos, devenu célèbre pour avoir été promu emblème de l'anonyme Français moyen, résiste plus à l'aimantation du sérieux, la perturbe par le surplomb paradoxal de sa posture.

Le sérieux ne se contente pas d'être moral; il peut aussi avoir des racines littéraires. Candidat idéal à la résistance tendancielle au «modernisme sceptique, réflexif et autoréférentiel ", le roman d'aventures occupe une place à part dans le discours critique, placé qu'il a été au centre d'une réflexion sur le roman au moment de l'une de ses crises ${ }^{\mathrm{xix}}$ - Grâce à Joseph Conrad et Robert L. Stevenson, on le savait propre à un sérieux paradoxal, malgré son origine populaire. L'aventure historique d'Anne et Serge Golon et de Christian Jacq et l'aventure moderne du thriller financier de Paul-Loup Sulitzer ont un sérieux documentaire, pédagogique, et même un sérieux idéologique (féminisme dans le cas des Golon, néo-libéralisme dans celui de Sulitzer). Ils campent toutefois bien près de la littérature populaire, avec leur fort tropisme sériel ; ce qui n'est plus le cas de la saga. Nonobstant la définition de la geste comme forme simple, autrefois avancée par André Jolles (1972 [1930]), une généalogie de la saga serait possible qui tiendrait compte des ambitions sociographiques du roman balzacien ou des rêveries génétiques de Zola, lourd héritage, convenons-en. Au moment de la crise post-naturaliste, un surgeon de ce réalisme s'était incarné dans une réinterprétation romanesque de la saga, bien éloignée de la geste médiévale, à partir des dix volumes de Jean-Christophe (1903-1912) de Romain Rolland. Ointe avec son auteur par le Nobel de 1915, cette forme devait connaitre son âge d'or entre-deux-guerres ${ }^{\mathrm{xx}}$. On constate que la saga romanesque ajoute souvent cette contrainte compositionnelle de la famille à un genre depuis plus longtemps, plus immédiatement et plus prolifiquement stabilisé dans l'histoire littéraire, le roman historique - un des grands favoris du territoire du milieu. Or, même si pour Jolles une forme primitive est un "geste verbal élémentaire", un ur-genre, un primitif de la narration, correspondant à une "disposition mentale » et si la disposition propre à la saga consiste en une interprétation de l'univers «en termes de clan, d'arbre généalogique, de liens du sang " (p. 64), rien n'empêche aussi de considérer la saga romanesque à partir de deux interprétants diamétralement opposés: soit comme la réalisation de cet élan totalisateur qui, selon Jacques Dubois (2000), dynamise l'esthétique réaliste soit une forme chic de la sérialisation, elle-même fortement caractéristique du roman populaire. Pas surprenant dès lors que dès la fin des années 40 semble s'opérer un transfert de cette formule romanesque depuis les Belles-lettres, où elle est en déclin, vers le territoire du milieu. En y adjoignant un terroir et sa forte thématisation territorialisante Gens de Mogador d'Élisabeth Barbier (1947) devait récupérer cette forme 
simple, la moyenniser en 6 tomes ${ }^{\mathrm{xxi}}$ et, sans doute en subissant l'attraction des séries télévisées, Jacqueline Monsigny, Claude Poulain, Charles Exbrayat, Florence Mothe, mais surtout Maurice Denuzière et Régine Deforges, allaient exploiter le plein potentiel de la formule, à partir des années $70^{\text {xxii }}$ - sorte de réaction de fond à l'immédiateté, au récit de l'actualité, denrées de base du régime médiatique.

Enfin, la cure de sérieux ne s'applique pas seulement aux contenus romanesques mais aussi aux politiques éditoriales. En ce cas, la moyennisation peut s'obtenir par deux dynamismes contraires. C'est coincé par le développement de Gallimard et Grasset entredeux-guerres qu'Albin Michel misait sur des écrivains goncourisables et des livres grand public comme le fidèle Maxence Van der Meersh ou le plus volage Henri Pourrat ${ }^{\text {xxiii. À }}$ l'inverse, c'est un processus de gentrification de la sérialité paralittéraire qu'a tenté le Fleuve noir avec sa collection "Grands Romans» (1954-1972). En ce cas, comme ses volumes in $-8^{\circ}$ distinguaient « Grands Romans » des collections en format poche de même que le prix, le format s'avérait déterminant. La politique du Fleuve noir pour ses collections sérielles avait mis l'accent sur des auteurs français alors que «Grands Romans » faisait la part belle aux traductions, surtout de l'anglais ${ }^{\text {xxiv }}$. Chacune des collections sérielles se spécialisaient dans un genre ; "Grands Romans » ne manifestait qu'un tropisme générique pour le roman d'aventures, exotiques ou historiques, le plus souvent tressé avec une intrigue amoureuse au détriment d'autres grandes familles de la narrativité populaire - le policier, le fantastique, la SF et les récits prospectifs... Malgré tous ces efforts, «Grands Romans » n'a toutefois pas réussi à dénier l'ADN du Fleuve noir : elle faisait appel à des auteurs-maison ${ }^{\mathrm{xxv}}$, à quelques gros fournisseurs ${ }^{\mathrm{xxv}}$, voire à des séries ${ }^{\mathrm{xxvi}}$, tout comme dans les collections sérielles.

En somme, le territoire du milieu considéré comme un champ de forces révèle la disparité des modes d'évaluation et la convergence que leur impose le régime médiatique, par la célébrité. Et il fait apparaître nombre de dynamismes mis en évidence par les voies du sérieux : moyennisation du roman mimétique par la casuistique, récupération de la saga tombée en désuétude dans la littérature highbrow, revendication du roman d'aventures qui avait d'abord largement été attaché la littérature populaire puis été "sauvé " par l'institution littéraire au début $\mathrm{du} \mathrm{XX}^{\mathrm{e}}$ siècle comme forme recevable de littérature légitime, abandon d'un format trop identificateur. Enfin, si l'on en croit les exemples qui se présentaient à l'esprit, même si l'affaire semble s'être nouée dans les années 1880 , elle s'est imposée lors de la montée en puissance de l'édition littéraire dans l'entre-deuxguerres - qu'accompagnaient parfois, comme chez Gallimard, des tensions intestines entre littérature d'élite et littérature payante ${ }^{\text {xxviii. }}$

\section{Juste bien}

Partir à la redécouverte de romans tombés dans l'oubli mais qui avaient eu du succès et élargir la connaissance du territoire du milieu de proche en proche par la redécouverte de romans apparentés à ces anciennes réussites fait prendre conscience de l'ampleur du territoire du milieu. Il comprendrait sans doute une très grande partie de la production française de fiction romanesque. Domaine si vaste qu'il a bien entendu déjà été partiellement décrit, même si ces descriptions restent régionales, incomplètes ou en attente de synthèse. Louable, l'objectif descriptif ne doit cependant pas justifier la simplification: ni en héroïsant la posture critique de redécouvreur (même si la désaffection pour les études littéraires rend forte la tentation), ni en réduisant trop 
hâtivement la complexité mise à jour ${ }^{\text {xxix }}$ ou la convergence et les divergences de dynamismes hétéroclites.

21 Après ces suggestions de méthode, resteraient à mentionner au moins trois points qui n'ont pas été abordés alors qu'ils méritaient de l'être. Primo, la traduction n'est apparue ici que de manière fugitive, allusive, alors que deux dossiers seraient à ouvrir sur la place du territoire du milieu dans les exportations de romans français subissant à la fois le test de la transculturalisation (laquelle peut s'effectuer sans changer de langue) et celui de la traduction et sur l'importance de la part des romans importés dans ce territoire en regard de ceux importés dans les genres sériels et chez les mimético-sceptiques.

Secundo, en partant d'une même situation de départ dans la culture de l'imprimé, contraster la situation française à la situation américain révèle souvent des trajectoires bien différentes - la BD née dans la littérature pour la jeunesse ici contre la BD née dans le journal làx ${ }^{\mathrm{xxx}}$, le magazine reconnaissant comme cible la consommatrice de classe moyenne dès 1900 là contre le magazine aboutissant au même objectif avec Marie-Claire en 1937 icixxxi. En quoi et comment la prétention à l'autonomie du champ littéraire en France a-t-elle fait diverger les trajectoires du roman middlebrow américain et du roman du territoire du milieu français? Les pièces issues du domaine français versées à ces premiers dossiers devraient enrichir la problématique de la république mondiale des lettres ${ }^{\mathrm{xxxii}}$.

Tertio, reconnaissons que pour s'être contentée d'une figuration statique, la tripartition de départ (le middlebrow, ni haute culture ni énergie du populaire) s'est avérée adéquate mais incomplète. Par le truchement d'une dernière métaphore faisant fonction d'hypothèse et avec souvenir des caractéristiques de la moyennisation selon Henri Mendras (1988) ${ }^{\mathrm{xxxiii}}$, cette tripartition ne serait-elle pas seulement le moment systolique de la moyennisation, une contraction évacuant le roman middlebrow du cœur de l'institution littéraire sous l'impulsion de telle ou telle valeur idéologico-esthétique tout en sachant qu'il ferait retour? Avec la domination du régime médiatique y aurait en effet succédé un moment diastolique avec son mouvement non plus centrifuge mais centripète : régime médiatique et processus de moyennisation sont devenus inséparables. Les romanciers expérimentateurs qui depuis le début du siècle dernier testaient les limites du genre, remettant en cause l'illusion référentielle, le statut de narrateur, la conscience du temps, le rôle de la description ou le contrat de lecture tout comme les romanciers qui insinuaient de l'invention dans les conventions de genres populaires se trouveraient désormais aspirés, mais dans un autre cœur. Là, sous l'effet d'une reconfiguration du lectorat, les frontières encore pertinentes au moment précédent tendraient à se brouiller - ce dont témoignerait par exemple l'abandon du format de poche par la Série noire, délaissant un format qui en avait longtemps été l'emblème : le polar se moyennise. Et, sans pour autant disparaître, l'institution littéraire n'aurait plus la même importance, remplacée par la célébrité médiatique ${ }^{\mathrm{xxxiv}}$ et le succès - marché, transmédiatisation, sérialisation... Au moment systolique on identifiait mal la fiction de culture moyenne, prise en étau entre la pleine reconnaissance du régime médiatique fondateur de la littérature populaire et sa dénégation par les Belles-lettres; au moment systolique, l'assurance impériale du territoire du milieu considèrerait d'un air protecteur les retardataires, prolétaires paralittéraires et aristocrates expérimentateurs. Le territoire du milieu? Un empire de la diversité foisonnante prise par des formes romanesques irriguées par un seul concept esthétique, le favori de mon coiffeur: «ni trop long, ni trop court, juste bien ». 


\section{BIBLIOGRAPHY}

\section{Études}

Casanova, Pascale. La République mondiale des lettres, Paris, Seuil, 1999.

Des Rivières, Marie-Josée \& Denis Saint-Jacques. » Le magazine en France, aux États-Unis et au Québec », in Production(s) du populaire, Jacques Migozzi \& Philippe Le Guern (sous la dir. de), 2004 , p. 29-37.

Dubois, Jacques. Les Romanciers du réel. De Balzac à Simenon, Paris, Seuil, coll. Points. Essais, 2000.

Fernandez, Ramon. « L'art de Conrad ». La Nouvelle Revue française, t. XXIII, n 135, décembre 1924, pp. 730-737.

Filon, Augustin. La Revue bleue, « Courrier littéraire », n² 23, 7 Déc. 1889.

Grasset, Pierre. «Le roman d'aventures, renouveau ou régression ? », La Grande Revue, vol. 108, juillet 1922, p. 605-620.

Jaloux, Edmond. « Joseph Conrad et le roman d'aventures anglais », t. XXIII, n 135, décembre 1924, p. 713-719.

Jolles, André. Formes simples, trad. Antoine Marie Buguet, Paris, Seuil, 1972 [1930].

Lacôte-Gabrysiak, Lylette. « » C'est un best-seller ! » », Communication, vol. 27, n 2, 2010, p. $187-216$.

Letourneux, Matthieu. Le roman d'aventures 1870-1930, Limoges, PULIM, coll Médiatexte, 2010.

Mendras, Henri. La Seconde Révolution française 1965-1984, Paris, Gallimard, 1988.

Mollier, Jean-Yves. L'Argent et les lettres. Histoire du capitalisme d'édition, 1880-1920, Paris, Fayard, 1988

Parinet, Élisabeth. Une Histoire de l'édition à l'époque contemporaine (XIX $\left.{ }^{e}-X X^{e} s.\right)$, Paris, Seuil, coll. Points. Histoire, 2004.

Pons, Christian-Marie \& Paul Bleton. Bande dessinée et figuration narrative, Québec, TÉLUQ, 1992.

Radway, Janice. A Feeling for Books : The Book-Of-The-Month Club, Literary Taste, and Middle-Class Desire, Chapel Hill, U. of North Carolina Press, 1999.

Ramsay, Arnaud. Jean d'Ormesson ou l'élégance du bonheur, Paris, Éd. du Toucan, 2009.

Rivière, Jacques. Le Roman d'Aventure, Paris, Édition des Syrtes, 2000 [1913].

Saint-Jacques, Denis, Jacques Lemieux, Claude Martin et Vincent Nadeau. Ces livres que vous avez aimés. Les best-sellers au Québec de 1970 à aujourd'hui, Québec, Nuit blanche éditeur, 1994.

Samoyault, Tiphaine. « Du roman-fleuve littéraire au roman-fleuve populaire : avatars de la série ", in De l'écrit à l'écran, sous la dir. de Jacques Migozzi, Limoges, PULIM, 2000, p. 271-280.

Tadié, Jean-Yves. Le roman d'aventures, Paris, Presses Universitaires de France, 1982. 
Thibaudet, Albert. « Réflexions sur la littérature : le roman de l'aventure », La Nouvelle Revue française, t. XIII, $\mathrm{n}^{\circ}$ 72, septembre 1919, p. 597-611 ; repris dans Réflexions sur le roman, Paris, Gallimard, 1938 [, p. 71-81.

Venayre, Sylvain. La Gloire de l'aventure. Genèse d'une mystique moderne, 1850-1940, Paris, Aubier, 2002.

Wittgenstein, Ludwig. Investigations philosophiques, trad. Françoise Dastur, Maurice Élie, Jean-Luc Gautero, Dominique Janicaud, Élisabeth Rigal, avant-propos et apparat critique Élisabeth Rigal, Paris, Gallimard, collection Bibliothèque de philosophie, 2005 [1953].

\section{Romans}

Armandy, André [André Albert d'Aguilard]. Rapa-Nui, Paris, Calmann-Lévy, 1923.

Armandy, André. Rapa-Nui, abondamment illustré par les photographies du film Société des cinéromans-films de France. Édition Pathé-Consortium. I. Un qui savait. Cinq qui voulaient. II. La Señorita Coreto, Paris, Jules Tallandier, 1928. 2 vol. In-8 à 2 col. t. 1, 80 p. ; t. 2, 76 p.

Barbusse, Henri. Le Feu. Journal d'une escouade, Paris, Flammarion, 1916.

Benoit, Pierre. L'Atlantide, Paris, Albin Michel, 1919.

Boudard, Alphonse. Les Combattants du petit bonheur, Paris, La Table ronde, 1977.

Cars, Guy des [Guy de Pérusse des Cars]. L'Officier sans nom, Cannes, impr. de Robaudy, s. d. [1941].

Cesbron, Gilbert. Chiens perdus sans collier, Paris, R. Laffont, 1954.

Cesbron, Gilbert. C'est Mozart qu'on assassine, Paris, R. Laffont, 1966.

Daninos, Pierre. Un Certain Monsieur Blot, Paris, Hachette, 1960.

Dekobra, Maurice. La Madone des sleepings, roman cosmopolite, Paris, Baudinière, 1925.

Hémon, Louis. Maria Chapdelaine, Paris, Bernard Grasset, 1924.

Hougron, Jean. La Nuit indochinoise : Tu récolteras la tempête, Paris, Donnat, 1950 ; Rage blanche, Donnat, 1951 ; Soleil au ventre, Donnat, 1952 ; Mort en fraude, Donnat, 1953 ; Les Portes de l'aventure, Donnat, 1954.

Kessel, Joseph. Le Lion, Paris, Gallimard, 1958,

Lanoux, Armand. Le commandant Watrin, Paris, Julliard, 1955.

Nicoidski, Clarisse. Couvre-feux, Paris, Ramsay, 1981.

Ohnet, Georges. Le Maître de forges, Paris, Paul Ollendorf, 1883.

Ormesson, Jean d'. La Gloire de l'Empire, Paris, Gallimard, 1971.

Vercel, Roger [Roger Delphin Auguste Crétin]. Capitaine Conan, Paris, A. Michel, 1934.

\section{Adaptations}

Andreani, Henri. L'homme qui assassina, sc. Firmin Gémier d'après Claude Farrère, avec, Firmin Gémier, Jean Toulout, Michelle, André Lefaur, 1913.

Bauer, Evgueny. Jizn za Jizn (Une vie pour une autre), d'après Georges Ohnet, avec Olga Rakhmanova, Vitold Polonskiï, Liliya Koreneva, Vera Kholodnaïa, 1916. 
Bernhart, Kurt \& Jean Tarride. L'homme qui assassina, sc. Heinz Goldberg, Henry Koster \& Harry Kahn, d'après Claude Farrère \& Pierre Frondaie, avec Jean Angelo, Marie Bell, 1933.

Bonnard, Mario. Rapa-Nui, sc. Franz Schulz, d'après André Armandy, avec Liane Haid, André Roanne, Claude Mérelle, Hans Albers, 1928.

Delanoy, Jean. Chiens perdus sans collier, sc. Jean Aurenche, Pierre Bost, François Boyer, d'après Gilbert Cesbron, avec Jean Gabin, 1955.

Farkas, Nicolas. La Bataille, sc. Bernard Zimmer et Nicolas Farkas d'après Claude Farrère, avec Charles Boyer, Viktor Tourjansky, Annabella, Valery Izkijineff, 1933.

Farkas, Nicolas. The Battle, sc. Bernard Zimmer et Nicolas Farkas d'après Claude Farrère, avec Charles Boyer, Viktor Tourjansky, Merle Oberon, Valery Izkijineff, 1933.

Fitzmaurice, George. The Right to Love, d'après Claude Farrère \& Pierre Frondaie, avec Mae Murray, David Powell, Holmes Herbert, 1920.

Gance, Abel. Le Maître de forges, d'après Georges Ohnet, avec Gaby Morlay, Henri Rollan, 1933.

Guillemot, Claude. La Brute, d'après Guy des Cars, avec Xavier Deluc, Assumpta Serna, Jean Carmet, 1987.

Majano, Anton Guilio, Il Padrone delle ferriere, d'après Georges Ohnet, avec Virna Lisi, Antonio Vilar, 1959.

O'Ferrall, George More. The Green Scarf, d'après Guy des Cars, avec Michael Redgrave, Ann Todd, Leo Genn, 1954.

Physioc, Wray B. Serge Panine, d'après Georges Ohnet, avec Laura La Varnie, William J. Butler, 1915.

Pouctal, Henri. Le Maître de forges ou Gerval le maître de forges, d'après Georges Ohnet, avec Gilbert Dalleu, 1912.

Méré, Charles (réal.). Serge Panine, Charles Méré et Paul Schiller (sc.), d'après Georges Ohnet, avec Françoise Rosay, Pierre Renoir, André Guize, Sylvia Bataille, 1938.

Rivers, Fernand. Le Maître de forges, sc. Abel Gance, Fernand Rivers, d'après Georges Ohnet, avec Hélène Perdrière, Jean Chevrier, 1948.

Rosenberg, Andréas. La Bataille, d'après Claude Farrère, France-Soir en septembre-octobre 1959.

Stora, Bernard. La Corruptrice téléfilm diffusé sur Antenne 2, d'après Guy des Cars, avec Catherine Hiegel, Samuel Labarthe, Laura Morante, 1994.

Tinayre, Daniel. Extraña ternura, d'après Guy des Cars, avec Egle Martin, José Cibriàn, 1964.

Tinayre, Daniel. Las Hermanas, d'après Guy des Cars, avec Myrtha Legrand, Silvia Legrand, Jorge Mistral, 1962.

Vecchiali, Paul, Philippe Morice, Susana Maria Rios Moore. L'Impure, téléfilm diffusé sur Antenne 2, sc. Jacques Espagne, Paul Vecchiali, d'après Guy des Cars, avec Mariane Basier, Ian Stewart Ireland, 1991.

Verneuil, Henri. Week-end à Zuydcoote, sc. François Boyer, d'après Robert Merle, avec Jean-Paul Belmondo, Catherine Spaak, Jean-Pierre Marielle, François Périer, Pierre Mondy, 1964.

Violet, Edouard Émile et Sessue Hayakawa, La Bataille, d'après Claude Farrère, avec Sessue Hayakawa, Felix Ford, 1923. 


\section{ENDNOTES}

i. Ex-tuteur du prince impérial Louis-Napoléon, fils de Napoléon III. Son précepteur-adjoint devait être Ernest Lavisse.

ii. C'est moi qui souligne.

iii. Plus tard, la troisième mouture par Alfred Vallette du Mercure de France (à partir de 1890), la Revue blanche des frères Natanson (1883-1904), la Nouvelle Revue Française de Charles-Louis Philippe puis Gaston Gallimard (à partir de 1908), etc.

iv. Cf. Jean-Yves Mollier (1988).

v. Conception qui la rend d'ailleurs un tantinet paradoxale en regard de l'argumentaire développé ultérieurement puisque l'ascèse sera un geste poïétique généralement rattaché à la culture légitime.

vi. La condamnation par un tribunal militaire d'un marin militant du PCF au motif qu'il avait invité ses camarades par des tracts à réclamer la cessation des hostilités en Indochine avait suscité une campagne contre sa condamnation à laquelle Jean-Paul Sartre avait participé par un livre.

vii. Au Livre de poche, au Fleuve noir et chez Pocket.

viii. Les deux éditions en deux volumes dans Select-Collection, en 1928 et 1935, le Livre de poche en 1988

ix. Collection des prix Goncourt des Éditions de l'Imprimerie nationale de Monaco, en 1950; collection Le Club des grands prix littéraires du Cercle du bibliophile, en 1966; collection Permanence des Éditions du Livre club Diderot, en 1968; collection Le Club des grands prix littéraires d'Édito-service, 1973...

x. Comme les deux volumes illustrés de gravures sur verre d'André Collot, publiés par J. Emery à Angoulême en 1949.

xi. Sur cette problématique, cf. Denis Saint-Jacques, Jacques Lemieux, Claude Martin et Vincent Nadeau (1994) et Christopher Todd (1994). J'utilise cet ouvrage comme prétexte ici pour ne pas développer ce point crucial plus avant.

xii. Par Henry Deyglun.

xiii. La Brute par Claude Guillemot en 1987 ; et, en téléfilms pour Antenne 2, L'Impure (1991) par Paul Vecchiali, Philippe Morice et Susana Maria Rios Moore, et La Corruptrice (1994) par Bernard Stora.

xiv. Respectivement en 1986 et 1988.

xv. Par Arnaud Ramsay en 2009.

xvi. C'est le Goncourt qui reste de loin le plus efficace en la matière, multipliant souvent les ventes de l'édition courante par un facteur 10 (suivi de loin par le Médicis et le Fémina, avec un facteur 4) et donnant un fort coup d'accélérateur aux rééditions en format poche, en distribution club et aux contrats de traduction. Lylette Lacôte-Gabrysiak (2010) évalue que, en moyenne entre 2005 et 2009, les Goncourt se sont vendus à 400000 exemplaires, les Renaudot à 198 000, les Femina à 156 000, les Goncourt des Lycéens à 132 000, les Prix des lectrices de Elle à 126000 , les Prix des Maisons de la presse à 87 000, les Interallié à 81 000, les Prix FNAC à 75 000, les Prix des libraires à 60 000, les Médicis à 55 000, les Prix du livre Inter à 55 000...

xvii. Tout best-seller ne relève pas du territoire du milieu (qu'on pense à San-Antonio) ni tout roman du territoire du milieu ne devient pas best-seller (qu'on pense à Dumur, bien des romans de Kessel ou Lartéguy).

xviii. À mettre en regard de la tendance exportatrice de romans français moyens vers d'autres langues. 
xix. Cf. Jacques Rivière (1913), Albert Thibaudet (1919), Pierre Grasset (1922), Ramon Fernandez (1924), Edmond Jaloux (1924). Pour un résumé du débat, cf. Jean-Yves Tadié (1982) ; et, avec une attention plus soutenue pour le discours social et le contexte historique, Sylvain Venayre (2002) et Matthieu Letourneux (2010).

xx. Avec les 16 volumes d'Histoire d'une société (1908-1924) de René Béhaine, les 9 des Thibault (1922-1940) de Roger Martin du Gard, aussi lauréat du Nobel en 1937, les 4 de L'âme enchantée (1924-1934) de Romain Rolland et des Hauts-Ponts (1932-1936) de Jacques de Lacretelle, les 27 des Hommes de bonne volonté (1932-1936) de Jules Romains, les 10 de la Chronique des Pasquier (1933-1944) de Georges Duhamel.

xxi. Trop virtuose du pastiche, Yves Gandon et son Pré aux dames (1947-1966) n'a sans doute pas bénéficié de toute la puissance virtuelle de la sérialisation puisque les 12 volumes de son ambitieuse « chronique romanesque de la société française » couvrant 7 siècles ont été dispersés chez différents éditeurs.

xxii. Cf. Tiphaine Samoyault (2000).

xxiii. Il publiait ses romans de la terre chez de nombreux éditeurs.

xxiv. Cela n'avait toutefois pas été le cas de la collection «Feu » à ses débuts. Incidemment, des auteurs parus dans "Grands Romans » avaient aussi été traduits dans «Feu », comme Geoffrey Atheling Wagner, Elleston Trevor.

xxv. G.J. Arnaud, Frédéric Dard et San-Antonio, Marc Arno, André Caroff, Serge Laforest, Claude Joste, Maurice Limat, Yves Dermèze, Pierre Nemours, Claude Rank, Richard-Bessière, Adam SaintMoore, dont peu de femmes, Mario Ropp, Giova Selly... Ce qu'il faudrait tempérer par le recours à des auteurs venus d'ailleurs, comme Pierre Mariel, ou des signatures connues dans le domaine du roman historique (Jacques Chabannes, André Besson, Yves Gandon), d'autres ne faisant que de brèves incursions dans les collections sérielles. Ainsi, Francis Murphy n'a-t-il contribué que pour un seul titre à la collection "Angoisse», contre quatre dans "Grands romans "; Paul-Joseph Marcel un roman policier contre une dizaine dans "Grands romans »; enfin quelques auteurs n'ont signé au Fleuve noir qu'un seul titre, dans cette collection (comme Jean-Marie Garraud).

xxvi. San-Antonio, G.J. Arnaud, Paul-Joseph Marcel, Michel Saint-Loup mais surtout Robert Gaillard.

xxvii. Comme celles de Jacques Chabannes, de Marie-Jacques Leygnac, de Robert Gaillard, ou, en traduction, la trilogie de Christopher Nicole.

xxviii. Cf. Élisabeth Parinet (2004).

xxix. Au-delà de l'intérêt descriptif du Book-of-the-Month Club dans A Feeling for Books (1999), audelà de l'idée selon laquelle les lectrices middlebrow sont sollicitées aussi bien dans le registre intellectuel que dans le registre émotionnel, c'est parce que Janice Radway fait apparaître une contradiction entre le principal argument de vente du club (le plaisir de la lecture) et l'évaluation (classification selon un système de valeurs éthiques et esthétiques) que son analyse est novatrice, même si son identification du plaisir de lecture au féminin et de la classification hiérarchisante et de compétition au masculin semble simplificatrice.

xxx. Cf. Christian-Marie Pons \& Paul Bleton (1992).

xxxi. Cf. Marie-Josée Des Rivières \& Denis Saint-Jacques (2004).

xxxii. Cf. Pascale Casanova (1999).

xxxiii. La mobilité comme valeur fondamentale, une dynamique de toupie accroissant tendanciellement les catégories intermédiaires et favorisant l'homogénéisation ; c'est-à-dire le contraire de la polarisation de la société.

xxxiv. Lylette Lacôte-Gabrysiak (2010) rapporte qu'à la question «Qu'est-ce qui vous incite le plus à acheter un livre?» d'un sondage Ipsos/Livre Hebdo de 2005, $57 \%$ des sondés avaient répondu « le bouche-à-oreille, les conseils de l'entourage », puis, en ordre décroissant, le prière d'insérer, les critiques dans la presse, les entrevues avec les auteurs - la personnalité de l'auteur ou son œuvre se frayant in fine une toute petite place. 


\section{ABSTRACTS}

The paper suggests ways to discuss the so-called middlebrow debates in the context of French literature. It attempts to understand it as a force field.

L'article pose les jalons de ce que la problématique du middlebrow pourrait être dans le domaine français. Il propose de le comprendre comme un champ de forces.

\section{INDEX}

Mots-clés: middlebrow, domaine français, territoire du milieu, institution littéraire, régime médiatique, célébrité, attractions centripète et centrifuge

\section{AUTHOR}

\section{PAUL BLETON}

Université Téluq, Montréal

Paul Bleton a publié des essais sur le roman d'espionnage (Les Anges de Machiavel, 1994, La Cristallisation de l'ombre, 2010), sur l'imaginaire français de la conquête de l'Ouest (Western, France, 2002) et sur l'acte de lecture propre au roman populaire (Ça se lit comme un roman policier, 1999). Une étude sur le récit de guerre et l'imaginaire militaire en France est à paraître et il prépare une histoire de l'adaptation du récit populaire (à la scène, à l'écran), depuis le mélodrame napoléonien à la fin du cinéma muet. Il a co-signé L'amour au temps du roman (2015) avec C. Caland et B. Rouby, Lignes de front. Le Roman de guerre africain (2009) avec D. Nyela et Le Vagabond stoïque, Louis Hémon (2004) avec M. Poirier. Il a dirigé plusieurs volumes collectifs Hostilités. Guerre, mémoire, fiction et culture médiatique (2001), Amour, aventure et mystère (1998), Les hauts et les bas de l'imaginaire western (avec Richard Saint-Germain, 1997), Armes, larmes, charmes (1995). Il a été le maître d'œuvre de plusieurs numéros thématiques de revues et publié de nombreux articles sur la fiction populaire. Professeur à l'Université Téluq à Montréal depuis 1982, il y a conçu des ouvrages pédagogiques de formation à distance sur le français langue seconde, la communication écrite et l'argumentation, la linguistique et la philosophie du langage, la communication non-verbale et la communication interculturelle, la littérature de grande diffusion et la BD. 